The Magnetic Moment of the Electron.

THE hypothesis of the spinning electron assigns to the electron an angular momentum $h / 4 \pi$ and a mag-

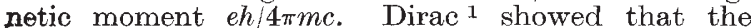
spectroscopic duplexity pointed out by Heisenberg can be explained more satisfactorily by modifying the wave equation. In his theory the angular momentum is still $h / 4 \pi$. The magnetic moment $\mu$, however, is only approximately the Bohr magneton $\mu_{0}$. Using Darwin's 2 explicit expressions for the four $\psi$ 's, the magnetic moment of the electron in the field of a charge $Z e$ is easily calculated. The result is

$$
\mu=\mu_{0}\left(1+2 \sqrt{1-a^{2} Z^{2}}\right) / 3,
$$

where $a$ is the fine structure constant $7.3 \times 10^{-3}$. Substituting $Z=92$ for uranium, we obtain $\mu=0 \cdot 83 \mu_{0}$.

Since in the nucleus there may be very intense fields, the conditions in it are approximated by using large values of $Z$. The highest value that may be used is 137 , because higher values make the solution inapplicable. For this $Z$ the moment decreases to $(1 / 3) \mu_{0}$. Performing the same calculation for excited states with radial quantum number $=0$, we find, using Darwin's notation and his first type of solution,

$$
\mu=\left(1+2 \sqrt{(k+1)^{2}-a^{2} Z^{2}}\right)(2 k+3)^{-1} \mu_{H},
$$

where $\mu_{H}$ is the ordinary magnetic moment of the state in question calculated neglecting relativity. The theoretically possible minimum of the above expression is $(2 k+3)^{-1} \mu_{H}$. Thus in intense fields such as may exist in the nucleus, the magnetic moment of the electron may be less than a Bohr magneton.

Dr. R. S. Mulliken pointed out to me that in several instances isotopes supposedly containing odd and even numbers of nuclear electrons have practically identical spectra. If the magnetic moment of the electron were always $\mu_{0}$ one of the two isotopes would have a resultant nuclear magnetic moment which would modify its spectrum very noticeably. Therefore he concluded that for the nuclear electrons $\mu$ may be much smaller than $\mu_{0}$. Dirac's theory is qualitatively at least in agreement with this conclusion of Mulliken. It shows that the electron spin can be modified by the presence of intense electric fields, and that in the cases mentioned above the magnetic moment is smaller than a Bohr magneton.

Barnett's ${ }^{3}$ and Emil Beck's ${ }^{4}$ measurements of the gyromagnetic ratio gave in contradiction to those of Chattock, Sucksmith, and Bates ${ }^{5}$ a value which indicates a somewhat smaller $\mu$ than would correspond to a Bohr magneton. There may be other reasons for this than the dependence of $\mu$ on the type and strength of the field. This may be, however, one of the reasons. A central field of the order of about $50 e$ corresponds to the observed deviation.

Department of Terrestrial Magnetism

G. Breit.

Carnegie Institution of Washington.

(Temporarily in Zurich.)

\section{The Depth of Field and Resolving Power of} Optical Instruments.

THE belief of some physicists that the significance of Airy's work of a hundred years ago on the character of optical images is rarely appreciated by users of optical instruments, will be strengthened by a recent letter in NATURE. It calls to mind a statement in Lord

\footnotetext{
1 P. A. M. Dirac, Proc. Roy. Soc., A, vol. 117, p. 610 ; vol. 118, p. 351 .

2 C. G. Darwin, idem, vol. 119.

S. J. Barnett, Proc. Amer. Acad, vol. 60, p, 127

Emil Beck, Ann. der Phys., vol. 60, p. 109.

5 Sucksmith and Bates, Proc. Roy. Soc., vol. 104, p. 499 ; vol. 108 ,
} p. 638 .

No. 3078, VoL. 122]
Rayleigh's address to the Royal Society in 1907: "In looking into the more recent progress of Geometrical Optics, I have been astonished to find how little correlation there has been. . . . In this subject it would appear that a man cannot succeed in making even his own countrymen attend to him." Lord Rayleigh himself appears to have fared no better than his great predecessors. More than forty years ago he discussed, in terms of the wave theory, the accuracy necessary in focussing, and verified his theory by experiment. Nevertheless, so far as I can recall, every book on optics or photography that deals with this question bases the discussion on the geometrical theory. The tables for finding depth of field published each year in the British Journal Photographic Almanac, and a recent contribution to NATURE, rest on the same assumptions.

Unfortunately, the geometrical method has a habit of returning the wrong answer to questions affecting the use of optical instruments. It leads us to expect an improvement in the definition given by a lens when the aperture is diminished, but the wave theory leads to the opposite conclusion. It declares that the depth of field should vary inversely as the first power of the diameter of the aperture, but the wave theory substitutes the second power. These two examples, out of many that might be cited, show the importance of considering optical questions in accordance with the concepts of the wave theory. The geometrical method, if employed at all, should only be used to find relations between loci where perfect ray convergence may be assumed. When tolerances are discussed by the wave method, it turns out that the quantities involved are invariants as found by this restricted geometrical method. The reason for this corre. spondence becomes clear when the wave theory is employed throughout ; it will be sufficient here to give examples.

If $y$ is a small length perpendicular to the axis, meeting it in the same point as a ray inclined at an angle $\theta$ with the axis, $\mu y \sin \theta$ is invariant on refraction if this elementary length is imaged without aberration. The parallel theorem states that two points in the same transverse plane will not appear distinct in the image if their separation $y$ is less than the value which satisfies $\mu y \sin \theta=\kappa \lambda$, where $\kappa$ is a constant and $\theta$ is the inclination to the axis of the extreme ray transmitted by the lens. If $x$ represents a small length measured along the axis of the lens, the invariance of $\mu x(1-\cos \theta)$ corresponds to a range of focus $x$, determined by the condition $\mu x(1-\cos \theta)=\kappa^{\prime} \lambda$, within which the minimum standard of definition depends on the constant $\kappa^{\prime}$. For sensibly perfect imagery $\kappa^{\prime}$ should not exceed $1 / 4$. In most photographic work values as high as 4 , or even 8 , are generally accepted as the equivalent of good definition. Except with high power microscope lenses, sin $\theta$ and $1-\cos \theta$ may be replaced by $a / 2 u$ and $\alpha^{2} / 8 u^{2}$ respectively, where $a$ is the diameter of the aperture and $u$ is the distance from the lens of the point from which the elementary displacements $x$ and $y$ are made.

A third law of geometrical optics, which is in effect a combination of the two just mentioned, states that the longitudinal magnification is proportional to the square of the transverse magnification; in symbols, $x / \mu y^{2}$ is invariant. The related theorem is that the depth of field associated with the distinct rendering of points distant $y$ apart is $x$, where $\lambda x=K \mu y^{2}, K$ being a constant depending on the quality of definition considered satisfactory. Evidently $K=2 \kappa^{\prime} / \kappa^{2}$, and sensibly perfect definition, with the central diffraction dises of distinctly rendered points in contact, is attained with $K=1 / 3$.

In all these expressions $\lambda$ is the wave-length of the light forming the image, measured in the medium for 